\title{
The role of melatonin in pregnancies complicated by placental insufficiency: a systematic review
}

\author{
Ilaria Fantasia ${ }^{1}$, Sofia Bussolaro ${ }^{1}$, Tamara Stampalija ${ }^{1}$, and Daniel Rolnik ${ }^{2}$ \\ ${ }^{1}$ Institute for Maternal and Child Health, IRCCS "Burlo Garofolo", Trieste \\ ${ }^{2}$ Monash University
}

April 8, 2021

\begin{abstract}
Background: Pregnancies complicated by placental insufficiency are characterised by increased oxidative stress. It has been suggested that melatonin has antioxidant properties and contributes to the maintenance of placental homeostasis. Objective: To systematically review the available literature about melatonin in pregnancies complicated by placental insufficiency, specifically preeclampsia and fetal growth restriction, exploring three different aspects: 1) maternal melatonin levels; 2) expression and activity of melatonin placental receptors; 3 ) effects of maternal melatonin administration. Search strategy: PubMed (Medline) and Scopus were searched until December 2020. Selection criteria: Published literature in English language describing the role of melatonin in pregnancies complicated by placental insufficiency, with a specific focus on preeclampsia and fetal growth restriction. Data collection and analysis: Identified studies were screened and assessed independently by two authors. Data were extracted and compiled in a qualitative evidence synthesis. Main results: Pregnancies complicated by placental insufficiency show a significant reduction in maternal systemic serum melatonin levels and a significant reduction in the expression of placental melatonin receptors. Melatonin administration in pregnancy seems safe and may reduce oxidative stress. Conclusion: The circadian pattern of melatonin secretion seems to be altered in pregnancies complicated by placental insufficiency. This is reflected by lower production of melatonin, with consequent lower systemic and placental concentrations, and lower expression of melatonin receptors, thus reducing the local release of the indole and its autocrine function. Funding: None Keywords: Melatonin, Preeclampsia, Fetal growth restriction, Placental insufficiency, Review.
\end{abstract}

The role of melatonin in pregnancies complicated by placental insufficiency: a systematic review

Authors: Ilaria Fantasia*1, Sofia Bussolaro ${ }^{1,2}$, Tamara Stampalija ${ }^{1,2}$, Daniel L Rolnik ${ }^{3}$

1. Institute for Maternal and Child Health, IRCCS "Burlo Garofolo", Trieste, Italy

2. Department of Medicine, Surgery and Health Sciences, University of Trieste, Trieste, Italy

3. Department of Obstetrics and Gynaecology, Monash University, Melbourne, Australia

*Corresponding author

Ilaria Fantasia

Unit of Fetal Medicine and Prenatal Diagnosis

Institute for Maternal and Child Health - IRCCS "Burlo Garofolo"

Via dell'Istria 65/1

34137 Trieste, Italia

e.mail: ilaria.fantasia@burlo.trieste.it

Phone: 00393280922893 
Running title: Melatonin and placental insufficiency

\section{Abbreviations}

FGR: fetal growth restriction

MR: melatonin receptor

HDP: hypertensive disease of pregnancy

SNC: suprachiasmatic nuclei

AANAT: arylalkylamine $\mathrm{N}$-acetyltransferase

ASMT: acetylserotonin methyltransferase

PE: preeclampsia

sFlt-1: soluble fms-like tyrosine kinase 1

PlGF: placental growth factor

PAPP-A: pregnancy associated plasma protein

ROS: reactive oxygen species

\section{ABSTRACT}

Background: Pregnancies complicated by placental insufficiency are characterised by increased oxidative stress. It has been suggested that melatonin has antioxidant properties and contributes to the maintenance of placental homeostasis.

Objective: To systematically review the available literature about melatonin in pregnancies complicated by placental insufficiency, specifically preeclampsia and fetal growth restriction, exploring three different aspects: 1) maternal melatonin levels; 2) expression and activity of melatonin placental receptors; 3) effects of maternal melatonin administration.

Search strategy: PubMed (Medline) and Scopus were searched until December 2020.

Selection criteria: Published literature in English language describing the role of melatonin in pregnancies complicated by placental insufficiency, with a specific focus on preeclampsia and fetal growth restriction.

Data collection and analysis: Identified studies were screened and assessed independently by two authors. Data were extracted and compiled in a qualitative evidence synthesis.

Main results: Pregnancies complicated by placental insufficiency show a significant reduction in maternal systemic serum melatonin levels and a significant reduction in the expression of placental melatonin receptors. Melatonin administration in pregnancy seems safe and may reduce oxidative stress.

Conclusion: The circadian pattern of melatonin secretion seems to be altered in pregnancies complicated by placental insufficiency. This is reflected by lower production of melatonin, with consequent lower systemic and placental concentrations, and lower expression of melatonin receptors, thus reducing the local release of the indole and its autocrine function.

Keywords: Melatonin, Preeclampsia, Fetal growth restriction, Placental insufficiency, Review.

\section{Tweetable abstract}

Melatonin production is lower in pregnancies complicated by placental insufficiency.

\section{INTRODUCTION}

Pregnancy is a peculiar time of a woman's life where the body has to adapt to several changes in order to sustain fetal growth and wellbeing and to protect itself from the stress induced by the pregnancy status ${ }^{1}$. 
When such adaptive changes fail, the risk of complications and adverse pregnancy outcome is increased ${ }^{2-5}$. As in all mammals, the circadian rhythm, which reflects the normal light:dark cycle over 24 hours, was evolutionarily set to regulate all biological functions to ensure they are carried out at the proper time of the day, increasing the chance of survival ${ }^{6,7}$. The central master clock of the circadian rhythm is located in the suprachiasmatic nuclei $(\mathrm{SCN})$ of the hypothalamus, that communicates with the body through the production of molecules that act on peripheric clock genes ${ }^{8,9}$. Melatonin is the most known of these molecules and plays a crucial role in several important physiological functions, including the regulation of the sleep-wake cycle but also acting as an antioxidant, anti-inflammatory, anti-apoptotic and anti-tumoral agent ${ }^{10,11}$.

During pregnancy, there are two main pathways through which melatonin plays its functions: the systemic release has a role in programming the circadian rhythmicity of the near-term uterus, by acting synergically with oxytocin in delivery, and in promoting fetal development ${ }^{12,}{ }^{13}$; the local release exerts antioxidant functions with melatonin acting as a direct free radicals' scavenger and also through its stimulation of other antioxidant enzymes and the reduction of anti-angiogenic factors and pro-inflammatory molecules ${ }^{9,14}$. Therefore, the local production of melatonin seems to have an important role in preserving placental homeostasis and, as shown in animal models, oral supplementation with melatonin reduces blood pressure, increases the production of angiogenic factors, increases the uterine arterial flow and the total serum antioxidant capacity ${ }^{15,16}$. Such function could be helpful in placental insufficiency-related disorders, like preeclampsia (PE) and fetal growth restriction (FGR), characterised by increased oxidative stress and pro-inflammatory status ${ }^{1}$.

In consideration of these aspects, the effect of melatonin in pregnancy has been studied with the aim to explore systemic maternal melatonin levels in women with placental insufficiency-related disorders and the effect of maternal melatonin administration on such pregnancy complications. The aim of this systematic review is to summarize the scientific knowledge about melatonin in the human population with a specific focus on placental insufficiency, particularly PE and FGR.

\section{METHODS}

A comprehensive systematic review was performed to identify studies that evaluated the role of melatonin in pregnancies affected by placental insufficiency. The Preferred Reporting Items for Systematic Reviews and Meta-analysis (PRISMA) guidelines ${ }^{17}$ were followed in the report of this review. The study was registered with the International Prospective Register of Systematic Reviews database (PROSPERO registration number: CRD42021231936) ${ }^{18}$.

Study identification and selection

A systematic English literature search was conducted from inception until December 2020 in PubMed (Medline) and Scopus. The search strategy consisted of relevant Medical Subject Headings (MeSH) terms and keywords, including "melatonin", "preeclampsia/eclampsia", "placental insufficiency", "intrauterine growth restriction (IUGR)" " fetal growth restriction (FGR)" (S1). Research was done by single MeSH term in order to include all possible articles published on the topic. Reference lists of relevant articles were searched manually to identify papers not found using the electronic search.

Inclusion criteria of our research strategy were clinical trials and research articles on this topic. Study protocols, case reports, review articles, editorials, letters to the editor, in vitro studies, animal experimental studies, and conference proceedings/posters that did not appear as full text papers were not included in the review. The following data were extracted: authors, study title, year of publication, study period, objective, number of participants recruited, methods, pregnancy outcomes, and key results.

Study eligibility assessment, data extraction and methodological quality assessment of the included studies were completed by two independent investigators (SB, IF). Data from each eligible study were extracted without modification of original information onto custom-made data collection forms. Disagreements were resolved by consensus with a third reviewer (DLR).

To improve the synthesis and understanding of the different topics on this theme, the selected original 
articles were divided into three subgroups. The first group involves articles that assessed blood, salivary and/or urinary levels of melatonin in women with PE/FGR; the second group is formed by studies on placental tissue in women with PE/FGR; the third group refers to intervention studies in which melatonin was administered in a selected population of women with PE and/or FGR to evaluate pregnancy outcomes.

Quality assessment

The quality assessment of each included study was performed using Quality Assessment of Diagnostic Accuracy Studies (QUADAS-2) criteria $^{19}$ for observational studies of melatonin or placental receptor levels and using the Risk Of Bias In Non-randomized Studies - of Interventions (ROBINS-I) tool for intervention studies $^{20}$. With QUADAS-2, each study was assessed in four domains related to the risk of bias: patient selection; index test; reference standard; flow and timing. Each domain was categorized as "low risk", "high risk", or "some concerns" of bias if data regarding the domain were "reported and adequate", "reported but inadequate", and "not reported", respectively, and the first three domains were assessed with respect to applicability. With ROBINS-I, each study was assessed in seven domains related to the risk of bias according to the seven groups of assessment of the tool. Each domain was categorized as "low risk", "moderate risk", "serious risk", "critical risk" of bias, or "no information", with the help of a series of signalling questions aiming to facilitate the process. The overall judgement was then established on the basis of the rating of the individual domains. The Robvis tool web app ${ }^{21}$ was then used to visualise the risk-of-bias assessments creating "traffic-light plots" and weighted bar plots, after applying the two separate quality criteria (S2).

Statistical analysis

Results from the included studies will be pooled to obtain summary estimates of the mean difference and 95\% confidence interval of serum melatonin levels and expression of placental melatonin receptors between pregnancies affected by placental insufficiency and those unaffected. Estimates will be also pooled according to the baseline disease (PE or FGR). Meta-analysis will be carried out using random-effects models in the statistical software R using the packages meta and metafor. Between-study heterogeneity will be evaluated by assessment of the $\mathrm{I}^{2}$ statistic and the Cochrane $\mathrm{Q}$ test. If a meta-analysis will not be possible a qualitative synthesis will be reported.

\section{RESULTS}

\section{Search results}

After a systematic research of the available literature an overall number of 642 studies was found. After removal of duplicates, a total of 153 research studies were obtained through database search (103 articles about melatonin and PE/eclampsia and 50 articles about melatonin and FGR). Figure 1 shows the flow diagram of the study selection. Review studies (64 articles) and study protocols (five articles) were excluded, as well as all articles not suitable for study type (i.e. book chapters, conference proceedings) or topic (not related to the research) (23 articles). A further 49 articles were excluded because of reportingin vitro (15 articles) or animal studies (34 articles). Therefore, 12 manuscripts were included for the systematic review: eight articles about melatonin and PE/eclampsia and four about melatonin and FGR.

Risk of bias within studies according to QUADAS and ROBINS-I criteria

Ten observational studies were included in the review. In the "patient selection" domain, two studies were classified as high risk because the patients were selected randomly at different gestational ages ${ }^{22,23}$. The remaining eight studies were considered as low risk. In the "index domain", one study was classified as high risk because the gestational age of the included population was limited to the early first trimester of pregnancy

24 . The remaining nine studies were considered as low risk. In the "reference range" domain four studies were classified as "some "concerns" of bias because the definition of PE/FGR was not reported"22, 23, 25, 26. The remaining six studies were considered as low risk. In the "flow and timing" domain, all studies were considered as low risk (Figure S1). The two intervention studies identified ${ }^{12,27}$ demonstrated the same critical issues and were considered at serious risk of bias because of: absence of a protocol of randomisation 
("classification of interventions" risk of bias domain); absence of blinding at intervention ("measurement of outcomes" risk of bias domain) (Figure S2).

Due to inconsistent methods and reporting of estimates of the included studies, meta-analysis was not possible.

Description of the studies included

Maternal systemic melatonin levels in pregnancies affected by placental insufficiency

During pregnancy, melatonin production maintains a circadian rhythm with night-time levels significantly higher than day-time levels ${ }^{28}$. While keeping this circadian rhythm, the production of melatonin overall slightly decreases during the first and second trimesters, then increases reaching its peak values after 32 weeks' gestation, followed by a rapid fall after birth ${ }^{28-31}$. Some authors investigated if this pattern is preserved in pregnancies with placental insufficiency or if the oxidative stress characterising these pregnancies is reflected in a disruption of this pattern.

Overall, seven studies addressed the issue of melatonin levels in pregnancies complicated by placental insufficiency, five in respect to PE and two in respect to FGR.

The main characteristics and results of the included studies are summarised in Table S1.

In four studies, maternal circulating melatonin levels were significantly lower in patients with PE compared to controls: Nakamuraet al. found that patients with severe PE had significantly lower serum night-time melatonin levels than women with both mild $\mathrm{PE}$ and uneventful pregnancies at 32-36 weeks' gestation $(\mathrm{p}<0.01)$ and at any gestational age below 36 weeks $(\mathrm{p}<0.05)^{32}$; Bouchlariotou et al. found that nocturnal levels of melatonin were significantly lower in patients with $\mathrm{PE}$ at $>32$ weeks than in normal pregnancies $(48.4 \pm 24.7 \text { vs. } 85.4 \pm 26.9 \mathrm{pg} / \mathrm{mL}, \mathrm{p}<0.001)^{33}$; Zeng et al . found that, at disease presentation, circulating levels of melatonin were significantly reduced in women with PE $(\mathrm{p}=0.001)^{34}$; Shimada et al. found that salivary melatonin levels were significantly lower in complicated pregnancies than in healthy pregnancies at any time of the day assessed $(\mathrm{p}<0.001)^{22}$. Only one study reported higher melatonin levels in women with $\mathrm{PE}$ compared to healthy pregnancies $(29.4 \pm 1.9 \text { vs } 22.7 \pm 1.8 \mathrm{pg} / \mathrm{mL}, \mathrm{p}<0.001)^{26}$.

Two studies addressed the question of melatonin levels in pregnancies complicated by FGR alone: Berbets et al. found lower melatonin levels $(126.87 \pm 14.87$ vs. $231.25 \pm 21.56 \mathrm{pg} / \mathrm{mL}, \mathrm{p}<0.001)$ in singleton pregnancies complicated by FGR in the third trimester ${ }^{23}$; conversely, Ramiro-Cortijo et al. did not find differences in maternal serum levels at 9-11 weeks' gestation in twin pregnancies subsequently complicated by FGR ${ }^{24}$.

Placental melatonin receptors in pregnancies affected by placental insufficiency

Following the study of Lanoix et al. ${ }^{14}$ that showed the presence of MT1 and MT2 melatonin receptors in placenta of healthy pregnancies providing evidence of local hormone production, some authors started to investigate whether in pregnancies complicated by placental insufficiency there is a down regulation of these two receptors, responsible for lower systemic levels of melatonin. Overall, four studies addressed this issue, three in respect to PE and one in respect to FGR.

The main characteristics and results of the included studies are summarised in Table S2.

In 2012, Lanoix et al. ${ }^{35}$ demonstrated a lower expression of both receptors in eight placental tissue samples from women with PE $(\mathrm{p}=0.042)$ associated to higher placental levels of serotonin, the precursor of melatonin $(\mathrm{p}=0.0095)$. The mechanism behind this finding is likely related to the reduced placental gene expression $(\mathrm{p}<0.05)$ and activity $(\mathrm{p}<0.001)$ of the two enzymes responsible for the conversion of serotonin in melatonin, and both present in the chorionic villi, the AANAT (arylalkylamine N-acetyltransferase) and ASMT (acetylserotonin methyltransferase) enzymes. These data were confirmed by Zeng et al. ${ }^{34}$ who also reported significantly lower expression of MT1 receptors in 27 placentas of women with PE $(\mathrm{p}<0.05)$. Different results were reported by Yamamoto et al. ${ }^{25}$, who found higher expression of MT1 receptors in 20 placentas from women with hypertensive disorders of pregnancy $(\mathrm{p}<0.001)$, mainly in cases with gestational hypertension; 
however, their expression was lower in cases with $\mathrm{PE}$ and even more so in PE superimposed on chronic hypertension.

A reduced expression of MT1 and MT2 receptors in pregnancies complicated by FGR has been reported by Berbets et al. ${ }^{36}$. The optical density of both MT1 and MT2 receptors was lower in pregnancies complicated by FGR than in controls $(\mathrm{p}<0.001)$ and the difference in receptor expression was more evident in the apical than in the stromal part of the syncytiotrophoblast of FGR placentas $(\mathrm{p}<0.001)$.

\section{Effects of melatonin administration in pregnancies complicated by placental insufficiency}

To date, there are only two clinical trials on the effect of melatonin administration in women complicated by $\mathrm{PE}$ or FGR ${ }^{12,27}$. Although these were not double-blind placebo-controlled trials, they showed that melatonin therapy is safe for both the mother and the fetus. Moreover, in respect to PE, treatment with $10 \mathrm{mg} \times 3 /$ day of melatonin prolonged the interval from diagnosis to delivery by approximately six days $(\mathrm{p}<0.05)$, and fewer antihypertensive drugs were required to treat the disease compared to an historical cohort of controls $(\mathrm{p}<0.05)$. On the contrary, treatment with $4 \mathrm{mg} \times 2$ /day of melatonin from diagnosis to delivery in pregnancy complicated by FGR did not show an improvement in birth weight $(\mathrm{p}=0.8)$ but there is evidence of lower placental oxidative stress by lower placental concentrations of malondialdehyde (MDA), a marker of late stage lipide peroxidation $(\mathrm{p}=0.04)$.

The main characteristics and results of the study included are summarised in Table S3.

\section{DISCUSSION}

\section{Main findings}

The main findings of this systematic review are that in pregnancies complicated by placental insufficiency there is: firstly, a significant reduction in maternal systemic serum levels of melatonin and secondly, a significant reduction in the expression of placental melatonin receptors.

Strengths and limitation

To our knowledge, this is the first systematic review on the role of melatonin in pregnancies complicated by placental insufficiency. The main limitation is that the included studies had, frequently, small sample sizes, and were heterogenous in terms of design, type of population included, gestational age at assessment, site and timing of melatonin dosage, reason why a meta-analysis was not possible. This prevented us from doing a meta-analysis but, despite these aspects, the majority of studies obtained similar results when comparing uneventful pregnancies and pregnancies complicated by placental insufficiency. In those that did not find a significant association there were some flaws in the inclusion criteria. In the group of studies that evaluated circulating levels of melatonin, the only one reporting higher levels of the indole in pregnancies complicated by placental insufficiency was by Tranquilli et al.; however, besides the small sample size, they included also women with an altered circadian rhythm of blood pressure and not only women with a strict diagnosis of PE 26. Similarly, in the placental melatonin receptor group of study, the group of Yamamoto et al. found higher expression of MT1 receptor in placenta of pregnancies with HDP; however, if data are stratified according to the subgroup of hypertension, they were higher in cases with gestational hypertension, which is the milder form of the disease, while their expression was lower in cases with PE and even more so in PE superimposed on chronic hypertension ${ }^{25}$.

\section{Interpretation}

The importance of a normal circadian rhythm in pregnancy has been demonstrated by studies that showed that pregnant women working night shifts or exposed to jetlag were more likely to suffer adverse pregnancy outcomes such as miscarriage, preterm labour and low neonatal birth weight ${ }^{7}$. Moreover, the maintenance of a circadian rhythm is also essential for the health of the cardiovascular system and to reduce the rate of hypertension ${ }^{37}$. The effect of sleep disturbance pattern on maternal blood pressure and uterine perfusion has been addressed in the study by Tang et $a l . .^{38}$ that showed that maternal systolic, diastolic, and mean 
arterial blood pressure were significantly lower in women with longer duration and better quality of sleep, while poorer sleep quality was associated with higher resistance in the uterine arteries.

In pregnancies complicated by placental insufficiency, especially PE, there is evidence of an imbalance between the excessive production of reactive oxygen species (ROS) and defensive antioxidative mechanisms, such as the production of melatonin. The most acknowledged cause of an impaired placental development is an insufficient remodelling of the spiral arteries in the first trimester that leads to increased resistance in the uterine arteries ${ }^{39}$. This triggers a cascade of events resulting in placental dysfunction, ischemia-reperfusion damage, increased placental production of ROS, reduced production of angiogenic factors (such as placental growth factor $[\mathrm{PlGF}]$ and pregnancy associated plasma protein A [PAPP-A]) and increased production of antiangiogenic factors (soluble fms-like tyrosine kinase 1 [s-Flt1]) ${ }^{1,40,41}$. From this perspective, melatonin may play an important role as free radical scavenger, directly and through its numerous metabolites, but also stimulating the activity of antioxidant enzymes, such as glutathione peroxidase, in a circadian rhythm fashion ${ }^{9,42,43}$. The results from the group of Lanoixet al. ${ }^{14}$ showing that the placenta can synthetise melatonin through the presence of the AANAT and ASMT enzymes on the chorionic villi, suggested that the pineal gland is not the only source of melatonin. They also showed that the chorionic villi express the two main melatonin receptors, MT1 and MT2, through which melatonin can exert its functions by systemic and autocrine routes. The chorionic villi are formed by two major parts: the internal cytotrophoblast, which has proliferative and antiapoptotic functions, that fuses and forms the more external syncytiotrophoblast, which does not have the properties to regenerate itself and is more exposed to oxidative stress. Damages of the cytotrophoblast are therefore reflected in lower production of syncytiotrophoblast cells and placental damage. In vitro placenta studies showed that melatonin has the ability to stabilise the villous trophoblast by inhibiting apoptosis of the cytotrophoblast through its antioxidant properties and through its action on MT1 and MT2 receptors, increasing the regeneration of the syncytiotrophoblast ${ }^{44}$, and stabilising placental homeostasis. This aspect may be of paramount importance in pregnancies complicated by placental insufficiency characterised by a primary dysfunction of the trophoblastic unit. The presence of lower systemic and placental levels of melatonin as well as lower expression and function of placental receptors, as summarised in this review, may be the reflection of placental impairment, specifically of the chorionic villi unit. In vivo studies have shown that exogenous administrations of melatonin can effectively increase its placental levels, as reported by Okataniet al. ${ }^{45}$, who demonstrated higher melatonin concentration in chorionic villi of women who underwent chorionic villous sampling after being given an oral melatonin dose of $6 \mathrm{mg}$. Moreover, the activity of the antioxidant enzyme glutathione peroxidase was tested in chorionic homogenates between one and three hours after melatonin administration, and its activity increased significantly, with peak levels occurring at three hours $(\mathrm{p}<0.001)$. Therefore, oral melatonin administration effectively increases the placental concentration and antioxidant activity but whether oral melatonin administration can reduce the dysfunction of pregnancies with placental insufficiency is still to be determined.

Historically, the treatment for PE is the delivery of the baby with removal of the placenta. More recently, the introduction of effective screening programs able to identify women at high risk for PE followed by the daily administration of aspirin at a dose of $150 \mathrm{mg}$ at 11-14 weeks' gestation has been shown to reduce by $62 \%$ the rate of $\mathrm{PE}$ requiring delivery before 37 weeks' gestation ${ }^{46}$. However, aspirin administration is less effective for the prevention of late PE and FGR. In normal pregnancies, melatonin levels tend to be normal or lower in the first and second trimesters while they increase thereafter, reaching a peak after 32 weeks. This pattern possibly reflects the preparation of the pregnant uterus to parturition by being exposed to molecules that increase it sensitivity to the action of oxytocin ${ }^{47}$. However, it could also be the expression of the need to balance the increased oxidative stress and metabolic demands of a senescent placenta and a growing fetus. From this perspective, the increased production of melatonin in the third trimester could be a key factor for the maintenance of placental homeostasis towards the end of pregnancy.

Even though there are only two studies on the effect of melatonin administration in placental insufficiency, there is some evidence that the use of this indole in high-risk pregnancies could play a role in reducing oxidative stress, preserving placental function and stability, and improving fetal neurological development. ${ }^{12,27}$ Moreover, the results on melatonin administration are promising in terms of neonatal outcomes due 
to the antioxidant action of the indole: data on animal studies demonstrated that the daily intravenous administration of $6 \mathrm{mg}$ of melatonin in $14 \mathrm{FGR}$ induced ewes entailed an improvement of lipid peroxidation in the cortical white matter with an improvement of the organization and density of the white and grey matter and an axonal damage rejection evaluated by hematoxylin and eosin stain. However, larger prospective randomised trials are needed to establish the real effect of melatonin in these pregnancies, two of them currently ongoing: one evaluating the prophylactic role of melatonin in women at increased risk of PE 48 , and one evaluating the role of melatonin in promoting neurodevelopment in FGR infants ${ }^{49}$.

\section{CONCLUSION}

As reviewed herein, the physiological and circadian pattern of melatonin secretion seems to be altered in pregnancies complicated by placental insufficiency. This is reflected by lower production of melatonin, with consequent lower systemic and placental concentrations, and lower expression of melatonin receptors, thus reducing the local release of the indole and its autocrine function. Further studies need to address if melatonin administration in pregnancy could prevent or improve the outcome of pregnancies complicated by PE/FGR.

Acknowledgment: None.

Disclosure of interest: None.

Contribution to authorship: Ilaria Fantasia and Sofia Bussolaro were directly involved in the study eligibility assessment, data extraction, methodological quality assessment of the included studies and in the drafting of the manuscript. Daniel Rolnik and Tamara Stampalija were involved in the final revision of the manuscript.

Details of Ethics Approval: Not required.

S1. Search strategy in finding articles through Pubmed and Scopus.

S2. QUADAS-2 and ROBINS-I Assessment Tool.

Figure 1. Study flow diagram.

Figure S1. Risk of bias according to QUADAS tool.

Figure S2. Risk of bias according to ROBINS-I tool.

Table S1. Main characteristics and results of the studies included on systemic maternal melatonin levels in pregnancies complicated by placental insufficiency.

Table S2. Main characteristics and results of the included studies on placental expression of melatonin receptors.

Table S3. Main characteristics and results of the included studies on the effect of maternal administration of melatonin in pregnancies complicated by placental insufficiency.

\section{References}

1. Chiarello DI, Abad C, Rojas D, et al. Oxidative stress: Normal pregnancy versus preeclampsia. Biochim Biophys Acta Mol Basis Dis. 2020 Feb 1;1866(2):165354.

2. Reutrakul S, Zaidi N, Wroblewski K, et al. Sleep disturbances and their relationship to glucose tolerance in pregnancy. Diabetes Care. 2011 Nov;34(11):2454-7.

3. Okun ML, Schetter CD, Glynn LM. Poor sleep quality is associated with preterm birth. Sleep. 2011 Nov $1 ; 34(11): 1493-8$.

4. Ding XX, Wu YL, Xu SJ, et al. A systematic review and quantitative assessment of sleep-disordered breathing during pregnancy and perinatal outcomes. Sleep Breath. 2014 Dec;18(4):703-13. 
5. Carnelio S, Morton A, McIntyre HD. Sleep disordered breathing in pregnancy: The maternal and fetal implications. J Obstet Gynaecol. 2017 Feb;37(2):170-8.

6. Daan S, Aschoff J. Circadian contributions to survival. In: Vertebrate circadian systems. Springer; 1982. p. $305-21$.

7. Mahoney MM. Shift work, jet lag, and female reproduction. International journal of endocrinology. $2010 ; 2010$.

8. Salustiano EM, De Pinho JC, Provost K, Ruano R, Zugaib M. Maternal serum hormonal factors in the pathogenesis of preeclampsia. Obstet Gynecol Surv. 2013 Feb;68(2):141-50.

9. Reiter RJ, Tan DX, Korkmaz A, Rosales-Corral SA. Melatonin and stable circadian rhythms optimize maternal, placental and fetal physiology. Hum Reprod Update. 2014 Mar-Apr;20(2):293-307.

10. Zisapel N. New perspectives on the role of melatonin in human sleep, circadian rhythms and their regulation. Br J Pharmacol. 2018 Aug;175(16):3190-9.

11. Chuffa LGA, Lupi LA, Cucielo MS, Silveira HS, Reiter RJ, Seiva FRF. Melatonin promotes uterine and placental health: Potential molecular mechanisms. Int J Mol Sci. 2019 Dec 31;21(1):300. doi: 10.3390/ijms21010300.

12. Miller SL, Yawno T, Alers NO, et al. Antenatal antioxidant treatment with melatonin to decrease newborn neurodevelopmental deficits and brain injury caused by fetal growth restriction. J Pineal Res. 2014 Apr;56(3):283-94.

13. Wilkinson D, Shepherd E, Wallace EM. Melatonin for women in pregnancy for neuroprotection of the fetus. Cochrane Database Syst Rev. 2016 Mar 29;3(3):CD010527.

14. Lanoix D, Beghdadi H, Lafond J, Vaillancourt C. Human placental trophoblasts synthesize melatonin and express its receptors. J Pineal Res. 2008 Aug;45(1):50-60.

15. Lemley CO, Meyer AM, Camacho LE, et al. Melatonin supplementation alters uteroplacental hemodynamics and fetal development in an ovine model of intrauterine growth restriction. Am J Physiol Regul Integr Comp Physiol. 2012 Feb 15;302(4):R454-67.

16. Uzun M, Gencer M, Turkon H, Oztopuz RO, Demir U, Ovali MA. Effects of melatonin on blood pressure, oxidative stress and placental expressions of TNF $\alpha$, IL-6, VEGF and sFlt-1 in RUPP rat model of preeclampsia. Arch Med Res. 2017 Oct;48(7):592-8.

17. Shamseer L, Moher D, Clarke M, et al. Preferred reporting items for systematic review and meta-analysis protocols (PRISMA-P) 2015: Elaboration and explanation. BMJ. 2015 Jan 2;350:g7647.

18. International prospective register of systematic reviews [homepage on the Internet].

19. Whiting PF, Rutjes AW, Westwood ME, et al. QUADAS-2: A revised tool for the quality assessment of diagnostic accuracy studies. Ann Intern Med. 2011 Oct 18;155(8):529-36.

20. Sterne JA, Hernán MA, Reeves BC, et al. ROBINS-I: A tool for assessing risk of bias in non-randomised studies of interventions. BMJ. 2016 Oct 12;355:i4919.

21. McGuinness LA, Higgins JPT. Risk-of-bias VISualization (robvis): An R package and shiny web app for visualizing risk-of-bias assessments. Res Synth Methods. 2021 Jan;12(1):55-61.

22. Shimada M, Seki H, Samejima M, Hayase M, Shirai F. Salivary melatonin levels and sleep-wake rhythms in pregnant women with hypertensive and glucose metabolic disorders: A prospective analysis. Biosci Trends. 2016 Feb;10(1):34-41.

23. Berbets A, Koval H, Barbe A, Albota O, Yuzko O. Melatonin decreases and cytokines increase in women with placental insufficiency. J Matern Fetal Neonatal Med. 2019 Apr 25:1-6. 
24. Ramiro-Cortijo D, Calle M, Rodríguez-Rodríguez P, et al. Maternal antioxidant status in early pregnancy and development of fetal complications in twin pregnancies: A pilot study. Antioxidants (Basel). 2020 Mar 25;9(4):269. doi: 10.3390/antiox9040269.

25. Yamamoto Dde R, Yamamoto Lde R, Rocha LP, et al. Increase of placental sensitivity to melatonin and the alteration to its local synthesis in hypertensive syndromes in pregnancy. Hypertens Pregnancy. 2013 May;32(2):120-8.

26. Tranquilli AL, Turi A, Giannubilo SR, Garbati E. Circadian melatonin concentration rhythm is lost in pregnant women with altered blood pressure rhythm. Gynecol Endocrinol. 2004 Mar;18(3):124-9.

27. Hobson SR, Gurusinghe S, Lim R, et al. Melatonin improves endothelial function in vitro and prolongs pregnancy in women with early-onset preeclampsia. J Pineal Res. 2018 Oct;65(3):e12508.

28. Tamura H, Nakamura Y, Terron MP, et al. Melatonin and pregnancy in the human. Reprod Toxicol. 2008 Apr;25(3):291-303.

29. Tamura H, Takasaki A, Taketani T, et al. Melatonin and female reproduction. J Obstet Gynaecol Res. 2014 Jan;40(1):1-11.

30. Kivelä A. Serum melatonin during human pregnancy. Acta Endocrinol (Copenh). 1991 Mar;124(3):233-7.

31. Pang SF, Tang PL, Tang GW, Yam AW, Ng KW. Plasma levels of immunoreactive melatonin, estradiol, progesterone, follicle stimulating hormone, and beta-human chorionic gonadotropin during pregnancy and shortly after parturition in humans. J Pineal Res. 1987;4(1):21-31.

32. Nakamura Y, Tamura H, Kashida S, et al. Changes of serum melatonin level and its relationship to feto-placental unit during pregnancy. J Pineal Res. 2001 Jan;30(1):29-33.

33. Bouchlariotou S, Liakopoulos V, Giannopoulou M, et al. Melatonin secretion is impaired in women with preeclampsia and an abnormal circadian blood pressure rhythm. Ren Fail. 2014 Aug;36(7):1001-7.

34. Zeng K, Gao Y, Wan J, et al. The reduction in circulating levels of melatonin may be associated with the development of preeclampsia. J Hum Hypertens. 2016 Nov;30(11):666-71.

35. Lanoix D, Guérin P, Vaillancourt C. Placental melatonin production and melatonin receptor expression are altered in preeclampsia: New insights into the role of this hormone in pregnancy. J Pineal Res. 2012 Nov;53(4):417-25.

36. Berbets AM, Davydenko IS, Barbe AM, Konkov DH, Albota OM, Yuzko OM. Melatonin 1A and 1B receptors' expression decreases in the placenta of women with fetal growth restriction. Reprod Sci. 2020 Aug 17.

37. Hermida RC, Ayala DE, Mojón A, Fernández JR. Blunted sleep-time relative blood pressure decline increases cardiovascular risk independent of blood pressure level-the "normotensive non-dipper" paradox. Chronobiol Int. 2013 Mar;30(1-2):87-98.

38. Tang Y, Zhang J, Dai F, et al. Poor sleep is associated with higher blood pressure and uterine artery pulsatility index in pregnancy: A prospective cohort study. BJOG. 2020 Nov 3.

39. Papageorghiou AT, Christina K, Nicolaides KH. The role of uterine artery doppler in predicting adverse pregnancy outcome. Best practice \& research Clinical obstetrics \& gynaecology. 2004;18(3):383-96.

40. Cartwright JE, Whitley GS. Strategies for investigating the maternal-fetal interface in the first trimester of pregnancy: What can we learn about pathology? Placenta. 2017;60:145-9.

41. O'Gorman N, Wright D, Poon L, et al. Multicenter screening for pre-eclampsia by maternal factors and biomarkers at 11-13 weeks' gestation: Comparison with NICE guidelines and ACOG recommendations. Ultrasound in Obstetrics \& Gynecology. 2017;49(6):756-60. 
42. Wilking M, Ndiaye M, Mukhtar H, Ahmad N. Circadian rhythm connections to oxidative stress: Implications for human health. Antioxidants \& redox signaling. 2013;19(2):192-208.

43. Tan D, Reiter RJ, Manchester LC, et al. Chemical and physical properties and potential mechanisms: Melatonin as a broad spectrum antioxidant and free radical scavenger. Current topics in medicinal chemistry. 2002;2(2):181-97.

44. Lanoix D, Lacasse A, Reiter RJ, Vaillancourt C. Melatonin: The smart killer: The human trophoblast as a model. Mol Cell Endocrinol. 2012;348(1):1-11.

45. Okatani Y, Wakatsuki A, Shinohara K, Kaneda C, Fukaya T. Melatonin stimulates glutathione peroxidase activity in human chorion. J Pineal Res. 2001 May;30(4):199-205.

46. Rolnik DL, Wright D, Poon LC, et al. Aspirin versus placebo in pregnancies at high risk for preterm preeclampsia. N Engl J Med. 2017;377(7):613-22.

47. Sharkey JT, Puttaramu R, Word RA, Olcese J. Melatonin synergizes with oxytocin to enhance contractility of human myometrial smooth muscle cells. The Journal of Clinical Endocrinology \& Metabolism. 2009;94(2):421-7.

48. Hobson SR, Wallace EM, Kingdom JC, Hodges RJ. A randomized double-blinded placebo-controlled intervention trial of melatonin for the prevention of preeclampsia in moderate- and high-risk women: The MELPOP trial. Methods Mol Biol. 2018;1710:347-52.

49. Palmer KR, Mockler JC, Davies-Tuck ML, et al. Protect-me: A parallel-group, triple blinded, placebocontrolled randomised clinical trial protocol assessing antenatal maternal melatonin supplementation for fetal neuroprotection in early-onset fetal growth restriction. BMJ Open. 2019 Jun 22;9(6):e028243,2018-028243.

\section{Hosted file}

Figure1_Flow diagram PRISMA.pdf available at https://authorea.com/users/406658/articles/

517250-the-role-of-melatonin-in-pregnancies-complicated-by-placental-insufficiency-a-

systematic-review 